\title{
Isolation and characterization of a cDNA clone for the complete protein coding region of the $\delta$ subunit of the mouse acetylcholine receptor
}

\author{
(bacteriophage $\lambda$ gt10/sequence homology profiles/BC3H-1 cells)
}

\author{
Robert J. LaPolla*, Katharine Mixter Mayne ${ }^{\dagger}$, and Norman Davidson* \\ *Department of Chemistry and +Division of Biology, California Institute of Technology, Pasadena, CA 91125 \\ Contributed by Norman Davidson, August 20, 1984
}

\begin{abstract}
A mouse cDNA clone has been isolated that contains the complete coding region of a protein highly homologous to the $\delta$ subunit of the Torpedo acetylcholine receptor (AcChoR). The cDNA library was constructed in the vector $\lambda$ gt10 from membrane-associated poly $(A)^{+}$RNA from BC3H1 mouse cells. Surprisingly, the $\delta$ clone was selected by hybridization with cDNA encoding the $\gamma$ subunit of the Torpedo AcChoR. The nucleotide sequence of the mouse cDNA clone contains an open reading frame of $\mathbf{5 2 0}$ amino acids. This amino acid sequence exhibits $59 \%$ and $50 \%$ sequence homology to the Torpedo AcChoR $\delta$ and $\gamma$ subunits, respectively. However, the mouse nucleotide sequence has several stretches of high homology with the Torpedo $\gamma$ subunit cDNA, but not with $\delta$. The mouse protein has the same general structural features as do the Torpedo subunits. It is encoded by a 3.3-kilobase mRNA. There is probably only one, but at most two, chromosomal genes coding for this or closely related sequences.
\end{abstract}

The nicotinic acetylcholine receptor (AcChoR) is a postsynaptic integral membrane protein complex composed of four subunits, denoted $\alpha, \beta, \gamma$, and $\delta$, with gel electrophoretic molecular weights ranging from 40,000 to 65,000 . The receptor is very abundant in the electric organ of the ray Torpedo and it has been extensively characterized at the biochemical, functional, and sequence levels (1). The amino acid sequences of the four Torpedo subunits have been determined from nucleotide sequences of full-length cDNA clones (2-7).

Nicotinic AcChoRs occur in lower overall abundances per unit mass of tissue at the neuromuscular junctions of vertebrate striated muscle and in various muscle-like cell systems in culture. Vertebrate systems are of greater interest than Torpedo for most electrophysiological studies and for cell biological studies of assembly (see ref. 8). The subunits of the vertebrate receptor are similar in general properties to those of Torpedo, but they are clearly somewhat divergent at the amino acid sequence level (9).

Full-length cDNA clones for the $\alpha$ subunit of a bovine receptor and chromosomal genes for the $\alpha$ subunit of humans and chickens have been characterized $(10,11)$, as has a partial cDNA clone for the $\alpha$ subunit of the mouse AcChoR (12). As a further step toward the complete characterization of the vertebrate genes, we report here the isolation and characterization of a cDNA clone containing the complete proteinencoding region of the $\delta$ subunit of the mouse AcChoR.

\section{MATERIALS AND METHODS}

Preparation of Membrane-Associated Polysomal Poly(A) ${ }^{+}$ RNA. BC3H-1 mouse cells were grown and induced to undergo differentiation on reaching confluency so as to express

The publication costs of this article were defrayed in part by page charge payment. This article must therefore be hereby marked "advertisement" in accordance with 18 U.S.C. $\$ 1734$ solely to indicate this fact.
AcChoR as described (13). A membrane pellet enriched in membrane-bound polyribosomes was prepared by a modification of the method of Merlie et al. (14). Our procedure differed in some details, including omission of the nuclear separation step and use of emetine $\cdot \mathrm{HCl}$ in all isolation buffers to prevent ribosome run-off. The final membrane pellet was dissolved in $6 \mathrm{M}$ guanidine $\cdot \mathrm{HCl} / 20 \mathrm{mM}$ sodium acetate, pH 5/1 mM dithiothreitol (15). RNA was purified by four cycles of precipitation with ethanol $(0.55 \mathrm{vol})$ and redissolution in the same buffer. Poly(A) ${ }^{+}$RNA was isolated by two cycles of oligo(dT)-cellulose chromatography.

cDNA Cloning in $\lambda$ gt10. A cDNA library was prepared in the bacteriophage vector $\lambda \mathrm{gt} 10$ by a method devised by Charles Rice of this institution (personal communication), based in part on the procedure of Okayama and Berg (16). First strand synthesis was carried out with $10 \mu \mathrm{g}$ of the membrane-bound poly $(\mathrm{A})^{+}$RNA essentially as described (16) except that $\mathrm{dT}_{(12-18)}$ was used for priming. Four micrograms of actinomycin D and 40 units of human placental ribonuclease inhibitor were included in the $100-\mu$ l reaction volume. Second strand synthesis by replacement of RNA from $4 \mu \mathrm{g}$ of the RNA.DNA hybrid was carried out without addition of primer by adding Escherichia coli DNA ligase and polymerase I, ribonuclease $\mathrm{H}, \mathrm{dNTPs}$, and other components as described (16). Internal EcoRI sites in the resulting duplex cDNA were then protected by methylation with EcoRI methylase. The duplex cDNA was blunt-ended with T4 DNA polymerase and all four dNTPs. Two micrograms of phosphorylated EcoRI linkers (12-mers) was added with T4 DNA ligase. After digestion with EcoRI, free linkers and their fragments were removed by four cycles of precipitation from $2 \mathrm{M}$ ammonium acetate with $\mathbf{0 . 6} \mathrm{vol}$ of isopropyl alcohol at $25^{\circ} \mathrm{C}$. Approximately $2.4 \mu \mathrm{g}$ of the final cDNA preparation was then ligated to $4.6 \mu \mathrm{g}$ of $E c o R I-d i g e s t e d ~ \lambda g t 10$ DNA in $25 \mu$, using T4 DNA ligase. The reaction mixture was incubated at $15^{\circ} \mathrm{C}$ for $15 \mathrm{hr}$. Recombinant $\lambda$ gt10 DNA was packaged in vitro (ref. 17 , pp. 264-268). Packaged phage were plated on $E$. coli strains C600 (ref. 17, p. 504) to assess the proportion of recombinants in the library or on $\mathrm{C600}$ $\Delta H f l$ (provided by Carol Nottenburg of the University of California, San Francisco) for screening purposes. An unamplified library of approximately $1 \times 10^{6}$ recombinants was obtained.

Library Screening. We screened $1 \times 10^{5}$ recombinant plaques in duplicate by plaque filter hybridization (ref. 17, pp. 324-328) at a density of $2 \times 10^{4}$ recombinant phage per 150-mm (diameter) plate. Full length Torpedo cDNA clones, kindly provided by D. Noonan ( $\alpha$ subunit), T. Claudio ( $\beta$ and $\delta$ ), and S. Heinemann ( $\gamma$; see ref. 5), were nick-translated to specific activities of approximately $1.0 \times 10^{8} \mathrm{cpm} / \mu \mathrm{g}$. The hybridization solution contained $50 \%$ (vol/vol) formamide,

Abbreviations: AcChoR, acetylcholine receptor; kb, kilobase(s); bp, base pair(s). 
$0.9 \mathrm{M} \mathrm{NaCl} / 50 \mathrm{mM}$ sodium phosphate, $\mathrm{pH}$ 7.4/5 mM EDTA ( $5 \times$ SSPE) (ref. 17, p. 447 ), $0.1 \%$ Ficoll, $0.1 \%$ polyvinylpyrrolidone, $0.1 \%$ bovine serum albumin, $0.1 \%$ sarcosine, denatured salmon sperm DNA at $100 \mu \mathrm{g} / \mathrm{ml}$, poly(rA), poly(rC), and poly(rG) at $2 \mu \mathrm{g} / \mathrm{ml}$ each, and $1 \mu \mathrm{g}$ of labeled plasmid probe. Hybridization was carried out at $42^{\circ} \mathrm{C}$ for $48 \mathrm{hr}$. Filters were washed in $30 \mathrm{mM} \mathrm{NaCl} / 3 \mathrm{mM}$ sodium citrate $/ 0.05 \%$ sarcosine at $50^{\circ} \mathrm{C}$. Positive plaques were purified and rescreened at least three times.

\section{RESULTS AND DISCUSSION}

Source of mRNA. Differentiation of the myogenic nonfusing mouse cell line $\mathrm{BC} 3 \mathrm{H}-1$ results in the biosynthesis of relatively large amounts of AcChoR (13). An additional enrichment of AcChoR mRNA by a factor of about 5 is achieved by isolation of membrane-bound polysomes (14).

Screening the cDNA Library. The cDNA library was screened at moderate stringency with hybridization probes prepared from full-length cDNA clones for each of the four subunits of the Torpedo AcChoR. The $\alpha, \beta$, and $\gamma$ probes each identified between 10 and 25 apparent positive plaques. No positives were obtained with the Torpedo $\delta$ probe, even when screened under less stringent conditions [33\% (vol/ vol) formamide $/ 5 \times \mathrm{SSPE}, 42^{\circ} \mathrm{C}$. We chose to characterize first the mouse cDNA clones selected by using the Torpedo $\gamma$ probe.

Restriction Enzyme Analysis of the Mouse cDNA Clones. Five clones that gave strong signals with the Torpedo $\gamma$ probe in gel blots and contained inserts of length greater than 1.0 kilobase $(\mathrm{kb})$ were analyzed in detail. Their restriction endonuclease maps are shown in Fig. 1. Indicated on the figure for reference purposes are the sites of the initiator methionine and the terminator codon as determined by nucleotide sequence analysis (see Fig. 3). Several features bear noting. First, all five clones possess identical restriction maps in their coding regions, indicating they were derived from the same or closely related messages. Second, the inserts have widely different $3^{\prime}$ ends. Nucleotide sequence determination revealed A-rich regions in the coding strand of the $\lambda 58$ insert corresponding to the $3^{\prime}$ ends of $\lambda 46$ and $\lambda 53$. Oligo(dT) priming from A-rich regions in the mRNA could be responsible for this phenomenon. Third, the $5^{\prime}$ ends of all five inserts are within 33 nucleotides of the initiator ATG codon (determined by sequence analysis; data not shown). $\lambda 60$ extends the furthest upstream from the initiator ATG (33 nucleotides). The sequence of $\lambda 58$, the clone subjected to detailed sequence analysis, extends five nucleotides upstream of the ATG. $\lambda 53$ begins at position 7 on Fig. 3 and $\lambda 61$ begins at position 14 on Fig. 3. (None of these values includes the EcoRI linker sequence.) Fourth, the inserts from $\lambda 58$ and $\lambda 60$ have different restriction maps near their $3^{\prime}$ ends. RNA gel blot experiments suggest that this difference is due to a cloning artifact in the $3^{\prime}$ untranslated region of $\lambda 58$ (see Fig. 6 below).

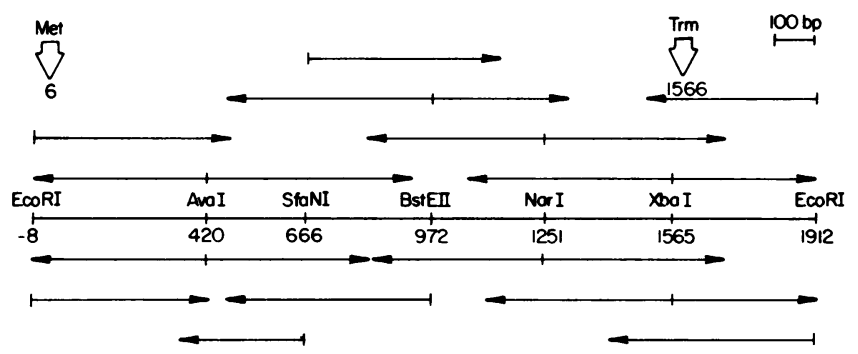

Fig. 1. Restriction maps of inserts from recombinant $\lambda$ cDNA clones. Positions of the initiator methionine (Met) and the termination codon (Trm) were determined from nucleotide sequence analysis (see Fig. 3). bp, Base pairs.

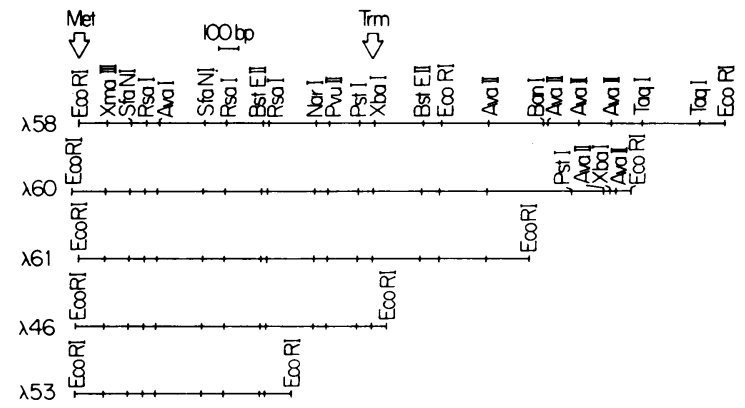

FIG. 2. Sequencing strategy for the protein-encoding region EcoRI fragment from $\lambda 58$ subcloned in pUC9. E. coli strain TB1 (provided by T. O. Baldwin, Texas A \& M University) was used for transformation. Plasmid DNA was isolated by the alkaline lysis/CsCl gradient method (ref. 17, pp. 90-91). The small closed arrows show the direction of sequencing. Protruding 5 ' ends were labeled by using T4 DNA kinase and $\left[\gamma^{32} \mathrm{P}\right] \mathrm{dATP}(>7000 \mathrm{Ci} / \mathrm{mmol}$, $\mathrm{ICN} ; 1 \mathrm{Ci}=37 \mathrm{GBq}$ ). Recessed 3 ' ends were labeled by using $E$. coli DNA polymerase I Klenow fragment (Bethesda Research Laboratories) and the appropriate $\alpha{ }^{32} \mathrm{P}$-labeled dNTPs $(\approx 3000 \mathrm{Ci} / \mathrm{mmol}$, Amersham). The vertical lines on the ends of the arrows indicate the position of the radioactive label. The nucleotide sequence was determined by the method of Maxam and Gilbert as modified by Smith and Calvo (19). The numbers indicate the position in nucleotides (from Fig. 3) of the restriction sites. The large open arrows show the locations of the initiator methionine (Met) and the termination (Trm) codons.

Nucleotide Sequence Determination. The EcoRI fragments in the five cDNA clones shown in Fig. 1 were subcloned separately in the plasmid vector pUC9 (18). Fig. 2 shows the strategy used to determine the nucleotide sequence of the EcoRI fragment from $\lambda 58$ that had been characterized as hybridizing to the coding region of the Torpedo AcChoR $\gamma$ subunit cDNA. Both strands of this fragment were completely sequenced.

Fig. 3 gives the nucleotide sequence of the coding (mRNA sense) strand of this fragment and the deduced amino acid sequence of the complete polypeptide chain. The first base of mouse cDNA from $\lambda 58$ is at position 1 of the nucleotide sequence in Fig. 3. The initiator ATG codon occurs at position 6. A signal peptide of 24 amino acid residues is encoded by nucleotides 6-77. The first amino acid of the mature protein begins at position 78 of the nucleotide sequence, as judged by comparison with the protein sequences of AcChoR subunits from fetal calf (9). There is an open reading frame of 1560 nucleotides (beginning at position 6), coding for a polypeptide chain of 520 amino acids and terminated by a TAG codon at position 1566 . There are two additional termination codons in phase with the reading frame at positions 1581 and 1605 . There is no sequence corresponding to the EcoRI linker at the $3^{\prime}$ end of this fragment, indicating that this EcoRI site is a natural internal EcoRI site in the mouse sequence. The calculated molecular weights of the mature polypeptide chain and its precursor are 57,104 and 59,393, respectively.

Sequence Comparisons. In Fig. 4 we have aligned the deduced amino acid sequence of the cloned mouse polypeptide chain with the amino acid sequences of the Torpedo AcChoR $\gamma$ and $\delta$ subunits. The similarity of the mouse amino acid sequence to the sequences of both of the Torpedo subunits is striking. The surprising result is that the mouse amino acid sequence is actually more similar overall to the Torpedo AcChoR $\delta$ subunit ( $59 \%$ homology) than it is to the Torpedo $\gamma$ subunit ( $50 \%$ homology). (In these calculations, gaps inserted into the sequences for alignment purposes are ignored by the computer.) When the individual amino acids were replaced by their functional groups (nonpolar, polar, acidic, and basic) and the same comparison made, the mouse poly- 


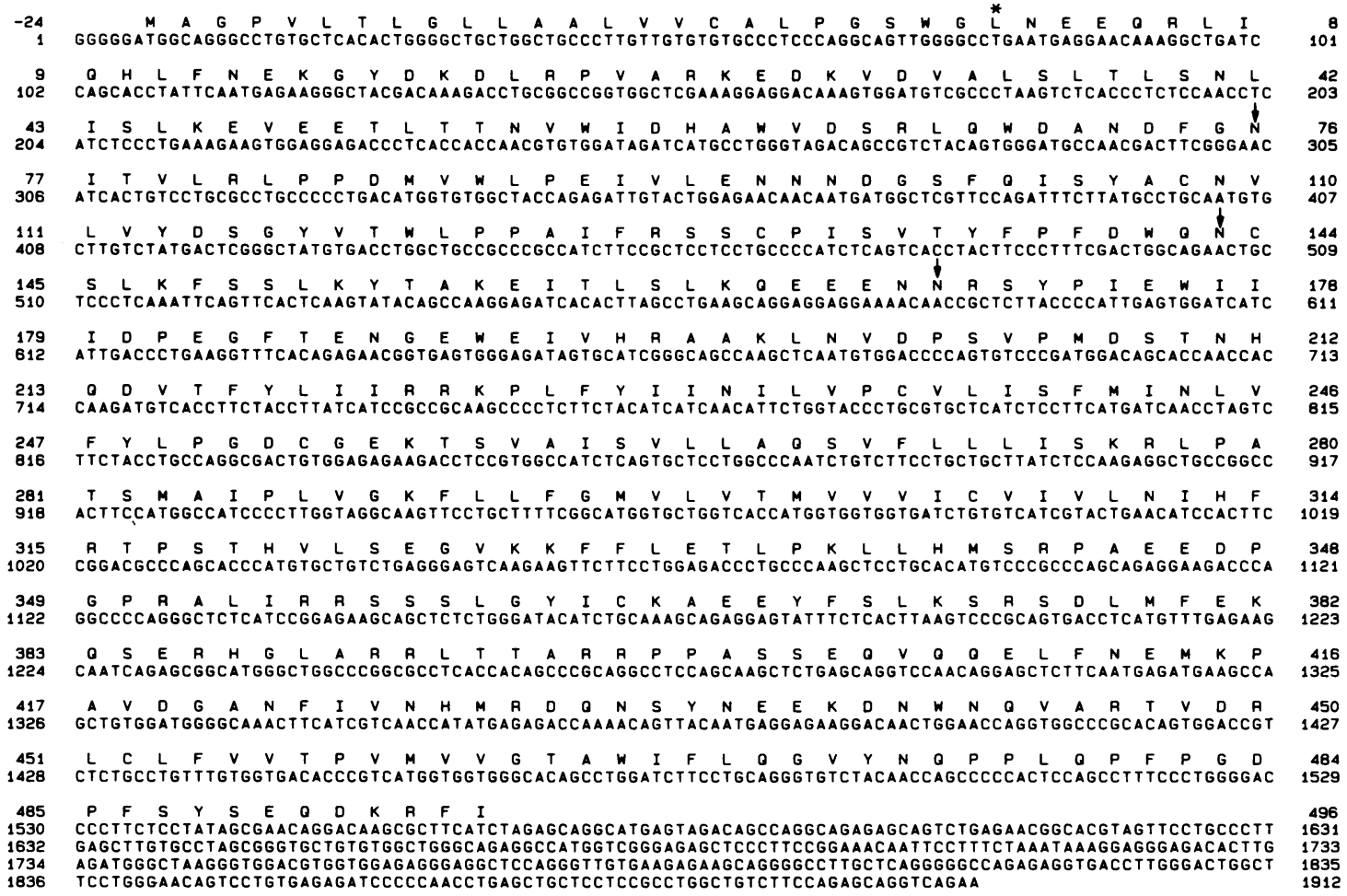

Fig. 3. Nucleotide sequence of the $E c o$ RI fragment from mouse cDNA clone $\lambda 58$ containing the protein coding region. The deduced amino acid sequence (standard one-letter code) of the open reading frame is given above the nucleotide sequence. Nucleotide no. 1 is the first nucleotide of the cDNA. Eight nucleotides d(A-A-T-T-C-G-G-G) present at the 5 ' end of the sequence were derived from the EcoRI linker used in the cloning procedure and have been deleted from the figure. Amino acid no. $1(*)$ is that assigned as the first amino acid of the mature protein. The arrows indicate potential sites of asparagine $\mathrm{N}$-glycosylation.

peptide chain was again more homologous overall to the Torpedo AcChoR $\delta$ subunit (77\%) than it was to the Torpedo $\gamma$ subunit $(72 \%)$. When the nucleic acid sequences were aligned as in Fig. 4, the mouse sequence was also more similar overall to the Torpedo $\delta$ subunit sequence $(60 \%)$ than it was to the Torpedo $\gamma$ subunit sequence (55\%) (data not shown). It is important to reiterate at this point that the Torpedo AcChoR $\gamma$ subunit cDNA was used to isolate this putative mouse AcChoR cDNA clone and that no positives were obtained when the Torpedo $\delta$ subunit cDNA was used as hybridization probe.

A clue to the cause for this paradox is provided by the percent homology profiles for both the nucleotide and amino acid sequences (Fig. 5). This analysis displays areas of high and low homology between two sequences as peaks and troughs, respectively. From the nucleic acid sequence comparisons it is apparent that the Torpedo $\gamma$ subunit cDNA sequence contains longer regions of high homology with the mouse cDNA sequence (Fig. 5A) than does the Torpedo $\delta$ subunit cDNA (Fig. $5 C$ ). We estimate that only sequences in the peaks of length $c a$. 75 nucleotides and homology greater than $65-70 \%$ in Fig. $5 A$ for the Torpedo $y$ cDNA probe would hybridize with the mouse sequence under the conditions used $\left(50 \%\right.$ formamide $\left./ 5 \times \operatorname{SSPE}, 42^{\circ} \mathrm{C}\right)$, whereas no such regions occur in the Torpedo $\delta \mathrm{cDNA}$.

In contrast to the nucleic acid homology profiles just described, the amino acid homology profiles show longer stretches of high homology for mouse vs. Torpedo $\delta$ than for mouse vs. Torpedo $\gamma($ Fig. $5 D$ and $B)$, in agreement with the overall percent homology calculations. These data indicate that the subunit of the mouse AcChoR that we have cloned is generally more similar to the Torpedo AcChoR $\delta$ subunit than to the $\gamma$ subunit. The nucleic acid sequence homology profiles explain the technical result of selecting a mouse $\delta$ cDNA clone with a Torpedo $\gamma$ probe; however, the biological and evolutionary significance of these homology relationships is unclear.

Conti-Tronconi et al. (9) have reported $\mathrm{NH}_{2}$-terminal amino acid sequence data for all four subunits of the fetal calf AcChoR. The data clearly showed sequence homology among the four subunits of the fetal calf AcChoR, similar to that observed in Torpedo and Electrophorus, and they established the subunit stoichiometry in fetal calf to be $2: 1: 1: 1$. However, the data did not permit subunit molecular weights

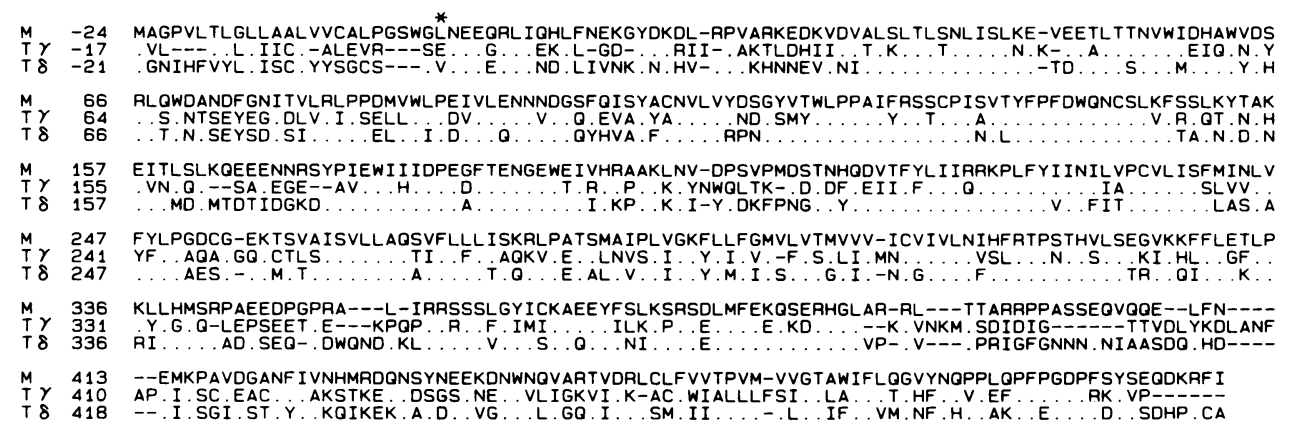

FIG. 4. Alignment of the ami65 no acid sequences of the mouse 156 (M) polypeptide and the Torpedo 156 (T) AcChoR $\gamma$ and $\delta$ subunits. The ${ }_{46}$ alignment of the latter is from 246 Noda et al. (4). Dots in the Torpedo sequences indicate identity 330 with the mouse sequence. Dashes indicate positions at which there 412 are no homologous amino acids. 417 In all three sequences, amino acid 496 no. $1(*)$ is the first amino acid of 501 the mature protein. 

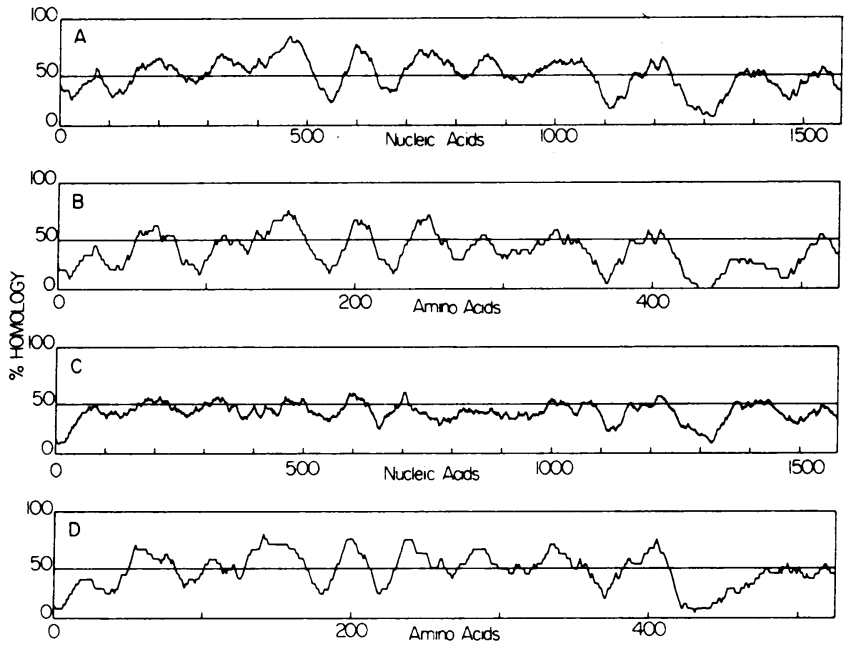

FIG. 5. Percent homology profiles for the mouse and Torpedo AcChoR subunits. In this analysis, the computer determines the percent homology between any two sequences (nucleic acid or amino acid) over a relatively short length of sequence (the search string). The computer begins the analysis at the $5^{\prime}$ or $\mathrm{NH}_{2}$ termini of the sequences to be compared and repeats the analysis over the entire length. A percent homology value is calculated over the search string for every residue in the sequences being compared. The nucleic acid sequence search string was 50 ; the amino acid sequence search string was 17 . Sequences were aligned as in Fig. 4. Only the protein-encoding regions of the nucleic acid sequences were compared. In this analysis, gaps inserted in the sequences for alignment purposes were scored as regions of $0 \%$ homology. $(A)$ Mouse vs. Torpedo $\gamma \mathrm{cDNA}$; $(B)$ mouse vs. Torpedo $\gamma$ amino acids; $(C)$ mouse vs. Torpedo $\delta \mathrm{cDNA} ;(D)$ mouse vs. Torpedo $\delta$ amino acids.

to be associated with two of the four sequences. The amino acid sequence deduced from the mouse cDNA clone described here shows a high degree of similarity to the $\mathrm{NH}_{2-}$ terminal amino acid sequence of the fourth subunit of the fetal calf AcChoR (labeled " $y$ " in ref. 9). Over the first 24 amino acids of the mature proteins, the sequences are identical at at least 12 residues. Many of the changes represent conservative substitutions.

As indicated by arrows in Fig. 3, there are three possible sites for $\mathrm{N}$-glycosylation, at asparagine residues 76,143 , and 169 of the mouse polypeptide. The site at position 143 is precisely conserved among mouse and all four Torpedo subunits. In both Torpedo $\gamma$ and $\delta$, there is a conserved site six amino acids toward the amino terminus relative to the mouse site at residue 76 .

The mouse polypeptide contains two cysteine residues at positions 130 and 144 which are also precisely conserved in all four subunits of the Torpedo receptor (2-5) and in the $\alpha$ subunits of the human and bovine receptors (10). These residues may be involved in disulfide bridge formation (2).

The calculated hydrophobicity profile (20) of the mouse subunit is very similar to the profiles calculated for the several Torpedo AcChoR subunits $(4,5)$. In particular, the lengths and positions of the several proposed hydrophobic membrane-spanning segments are highly conserved. For the mouse $\delta$ subunit the residue positions of these segments are M1, 225-251; M2, 257-275; M3, 291-312; and M4, 451-469 (data not shown).

Finer-Moore and Stroud (21) have proposed recently that all four subunits of the Torpedo AcChoR contain an amphipathic $\alpha$-helical membrane-spanning segment that constitutes part of the ion channel. The salient feature of this domain is that one side of the proposed $\alpha$-helix is composed predominantly of hydrophobic residues that interact with the other hydrophobic domains of the subunit polypeptides, whereas another side is composed of hydrophilic residues, which constitute part of the wall of the ion channel (21). To examine the possibility that such a domain may exist in this subunit of the mouse AcChoR, an $\alpha$-helical wheel was constructed beginning with residue 407 (Fig. 3) and ending with residue 428 . The overall average hydrophobicity (20) of this region was calculated to be -0.34 , a weakly hydrophilic value. The $\alpha$-helical wheel revealed a strongly hydrophilic surface on the putative $\alpha$-helix composed of residues 408,412 , $415,419,422$, and 426 (Fig. 3), whose average hydrophobicity was -3.57 . The average hydrophobicity of all the other residues in this region was 0.88 . This preliminary analysis suggests that an amphipathic domain may exist in this subunit of the mouse AcChoR as well.

RNA Blot Analysis. Fig. $6 A$ shows an RNA gel blot with membrane-associated poly(A) ${ }^{+}$RNA from BC3H-1 cells probed by hybridization with subcloned EcoRI fragments from three of the $\lambda$ recombinants (see Fig. 1). When the coding region subclone $6 \mathrm{H}$ was used to probe RNA from differentiated $\mathrm{BC} 3 \mathrm{H}-1$ cells, a predominant band at $3.3 \mathrm{~kb}$ was observed. Weaker bands at 4.6 and $2.0 \mathrm{~kb}$ were also detected. The major RNA band at $3.3 \mathrm{~kb}$ probably represents the mRNA species encoding this subunit of the mouse AcChoR.

The nature of the bands at 4.6 and $2.0 \mathrm{~kb}$ is not known. The band at $4.6 \mathrm{~kb}$ could represent a partially spliced precursor of the 3.3-kb species. (Nuclei were not removed from the cell homogenate prior to preparation of the membrane pellet.) The band at $2.0 \mathrm{~kb}$ could be a messenger related by sequence to the coding region of the mouse AcChoR $\delta$ subunit.

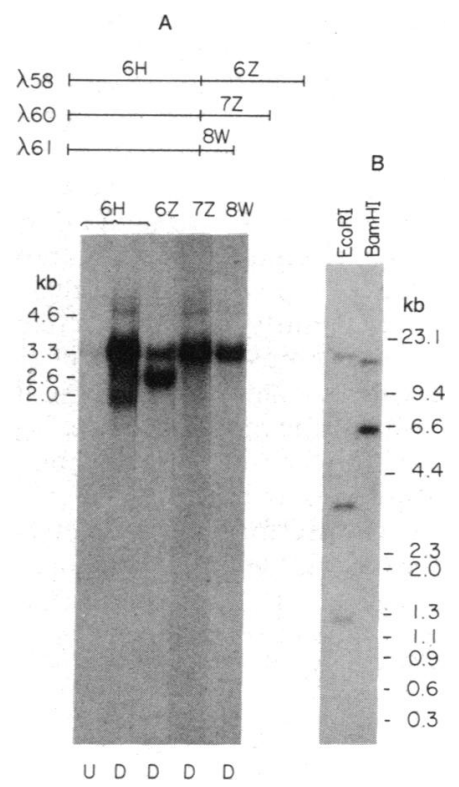

Fig. 6. (A) RNA blot analysis of mouse membrane poly(A) ${ }^{+}$ RNA. RNA was prepared from differentiated $B C 3 H-1$ cells (D). Undifferentiated RNA (U) was prepared from BC $3 \mathrm{H}-1$ cells harvested after 3-4 days of growth in $20 \%$ fetal calf serum. Poly(A) ${ }^{+}$RNA was electrophoresed through a $1.5 \%$ agarose gel containing $2.2 \mathrm{M}$ formaldehyde and then transferred to nitrocellulose paper (see ref. 22). Nick-translated EcoRI fragments $6 \mathrm{H}, 6 \mathrm{Z}, 7 \mathrm{Z}$, and $8 \mathrm{~W}$ (each subcloned in pUC9) were used as hybridization probes. (Subclone $6 \mathrm{H}$ contains the protein-encoding region.) Hybridization was carried out as described (22), except that dextran sulfate was omitted. Length standards were denatured HindIII-digested $\lambda$ DNA and Hae III-digested $\phi$ X174 DNA. (B) Gel blot analysis of mouse genomic DNA probed by hybridization with the EcoRI fragment from $\lambda 58$ containing the protein-encoding region. BALB/c mouse genomic DNA $(12 \mu \mathrm{g})$ was digested exhaustively with EcoRI or BamHI and run on a $0.7 \%$ agarose gel. The DNA was transferred to nitrocellulose and hybridized to nick-translated mouse cDNA (see ref. 17, pp. 383-389). Length standards were HindIII-digested $\lambda$ DNA and Hae III-digested $\phi$ X174 DNA. 
It is tempting to speculate that the $2.0-\mathrm{kb}$ RNA could be the message for the $\gamma$ subunit of the mouse AcChoR, although there is no evidence at this time to support this conjecture.

In gel blot analysis of membrane-bound poly $(\mathrm{A})^{+}$RNA from undifferentiated $\mathrm{BC} 3 \mathrm{H}-1$ cells the intensity of hybridization to the 3.3-kb RNA is about $1 / 50$ that for differentiated cells, confirming that expression of this subunit is induced by differentiation.

All three $3^{\prime}$ untranslated region subclones $(6 \mathrm{Z}, 7 \mathrm{Z}, 8 \mathrm{~W})$ also hybridized to the 3.3-kb RNA band. However, subclone $6 \mathrm{Z}$ also hybridized intensely to a band at $2.6 \mathrm{~kb}$ not detected with any of the other probes. This result suggests that a cloning artifact has occurred in the $3^{\prime}$ EcoRI fragment of the $\lambda 58$ insert. The fact that the restriction maps of the $\lambda 58$ and $\lambda 60$ inserts diverge after the first (5') Ava II site suggests that, somehow in the course of cloning, an unrelated piece of cDNA became attached to the $3^{\prime}$ end of the $\lambda 58$ insert at a point between this Ava II site and the Ban I site (see Fig. 1).

Genome Blot Analysis. To obtain some insight into the number of genes coding for this subunit of the mouse AcChoR, the genome blot shown in Fig. $6 B$ was prepared. Multiple genes for one or more subunits of the mammalian AcChoR may explain the observed kinetic and drug sensitivity differences among AcChoRs at the neuromuscular junction and those in other parts of the nervous system. The simple pattern of hybridization obtained (three bands in the EcoRI lane and two in the BamHI lane) is consistent with probably only one and at most two genes for this subunit of the mouse nicotinic AcChoR.

Further Discussion. We have isolated and sequenced a cDNA clone coding for a subunit of the mouse AcChoR. Comparison of this sequence with the sequences for Torpedo and other species confirms the general high degree of amino acid sequence conservation among the several AcChoR subunits in evolution. Although the mouse clone was selected by hybridization to a Torpedo $\gamma$ subunit cDNA probe, amino acid sequence comparison indicates a slightly higher degree of homology with the Torpedo $\delta$ subunit than with $\gamma$. On this basis, we tentatively identify our clone as that of the mouse $\delta$ subunit. We note that at present the only distinguishing criterion between Torpedo $\gamma$ and $\delta$ subunits is the small difference in gel electrophoretic mobility. A decisive functional or structural criterion for the role of each subunit in the assembled AcChoR awaits discovery.

Just when this manuscript was completed, we learned of the work of Nef et al. (23) with full sequence information on a genomic clone coding for the chicken AcChoR $\gamma$ and $\delta$ subunits. Our mouse amino acid sequence is more similar to that assigned by these authors to chicken $\delta$ than to that for chicken $\gamma$.

We are grateful to Dr. Dan Noonan for much help and advice and for providing a Torpedo $\alpha$ cDNA clone, to Dr. Toni Claudio for Tor- pedo $\beta$ and $\delta$ clones, and to Dr. Steve Heinemann for the Torpedo $\gamma$ clone (5). Dr. J. P. Merlie provided the BC3H-1 cells. Dr. Barbara Wold provided Agt10 DNA; she and Dr. Charles Rice contributed helpful advice about the cDNA cloning procedure. Tim Hunkapiller provided valuable assistance in the computer analysis of the data. This research has been supported by research grants to N.D. from the National Institutes of Health and the Muscular Dystrophy Association, by a National Institutes of Health Fellowship to R.L.P., and by a National Institutes of Health Predoctoral Traineeship to K.M.M.

1. Conti-Tronconi, B. M. \& Raftery, M. A. (1982) Annu. Rev. Biochem. 51, 491-530.

2. Noda, M., Takahashi, H., Tanabe, T., Toyosato, M., Furutani, Y., Hirose, T., Asai, M., Inayama, S., Miyata, T. \& Numa, S. (1982) Nature (London) 299, 793-797.

3. Noda, M., Takahashi, H., Tanabe, T., Toyosato, M., Kikyotani, S., Hirose, T., Asai, M., Takashima, H., Inayama, S., Miyata, T. \& Numa, S. (1983) Nature (London) 301; 251-255.

4. Noda, M., Takahashi, H., Tanabe, T., Toyosato, M., Kikyotani, S., Furutani, Y., Hirose, T., Takashima, H., Inayamà, S., Miyata, T. \& Numa, S. (1983) Nature (London) 302, 528-532.

5. Claudio, T., Ballivet, M., Patrick, J. \& Heinemann, S. (1983) Proc. Natl. Acad. Sci. USA 80, 1111-1115.

6. Sumikawa, K., Houghton, M., Smith, J. C., Bell, L., Richards, B. M. \& Barnard, E. A. (1982) Nucleic Acids Res. 10, 5809-5822.

7. Devillers-Thiery, A., Giraudat, J., Bentaboulet, M. \& Changeux, J.-P. (1983) Proc. Natl. Acad. Sci. USA 80, 2067-2071.

8. Merlie, J. P. (1984) Cell 36, 573-575.

9. Conti-Tronconi, B. M., Gotti, C. M., Hunkapiller, M. W. \& Raftery, M. A. (1982) Science 218, 1227-1229.

10. Noda, M., Furutani, Y., Takahashi, H., Toyosato, M., Tanabe, T., Shimizu, S., Kikyotani, S., Kayano, T., Hirose, T., Inayama, S. \& Numa, S. (1983) Nature (London) 305, 818-823.

11. Ballivet, M., Nef, P., Stalder, R. \& Fulpius, B. (1984) Cold Spring Harbor Symp. Quant. Biol. 48, 83-87.

12. Merlie, J. P., Sebbane, R., Gardner, S. \& Lindstrom, J. (1983) Proc. Natl. Acad. Sci. USA 80, 3845-3849.

13. Patrick, J., McMillan, J., Wolfson, H. \& O'Brien, J. C. (1977) J. Biol. Chem. 252, 2143-2153.

14. Merlie, J. P., Hofler, J. G. \& Sebbane, R. (1981) J. Biol. Chem. 256, 6995-6999.

15. Strohman, R. C., Moss, P. S., Micou-Eastwood, J., Spector, D., Przybyla, A. \& Paterson, B. (1977) Cell 10, 265-273.

16. Okayama, H. \& Berg, P. (1982) Mol. Cell. Biol. 2, 161-170.

17. Maniatis, T., Fritsch, E. F. \& Sambrook, J. (1982) Molecular Cloning: A Laboratory Manual (Cold Spring Harbor Laboratory, Cold Spring Harbor, NY).

18. Vieira, J. \& Messing, J. (1982) Gene 19, 259-268.

19. Smith, D. R. \& Calvo, J. M. (1980) Nucleic Acids Res. 8, 2255-2274.

20. Kyte, J. \& Doolittle, R. F. (1982) J. Mol. Biol. 157, 105-132.

21. Finer-Moore, J. \& Stroud, R. M. (1984) Proc. Natl. Acad. Sci. USA 81, 155-159.

22. Fyrberg, E. A., Mahaffey, J. W., Bond, B. J. \& Davidson, N. (1983) Cell 33, 115-123.

23. Nef, P., Mauron, M., Stalder, R., Alliod, C. \& Ballivet, M. (1984) Proc. Natl. Acad. Sci. USA 81, 7951-7999. 